\title{
EEDTCA: Energy Efficient, Reduced Delay and Minimum Distributed Topology Control Algorithm for Mobile Ad Hoc Network
}

\author{
Jayanthi Vagini K \\ Shiro Software Solutions \\ Nagercoil, T.N., India
}

\author{
Hemalatha M \\ Hindustan College of Arts and \\ Science, Coimbatore \\ T.N., India
}

\author{
Dinesh R \\ Shiro Software Solutions \\ Nagercoil, T.N., India
}

\begin{abstract}
Processing packets across Mobile Ad hoc Network, Topology control minimize interference among node in a network, increase the network capacity and extend lifetime of the network. Emerging research in mobile ad hoc networks (MANETs) says, there is a growing requirement of quality of service $(\mathrm{QoS})$ in terms of delay. In order to resolve the delay problem, it is essential to consider topology control in delay constrained environment with energy efficient. In this paper, we discuss reduced delay and minimum distributed topology control algorithm for mobile ad hoc networks. In this proposed system, we study on the delay-constrained topology control problem, and take into account delay and energy efficiency. Simulation results are presented demonstrating the effectiveness of this new technique as compared to other approaches to topology control.
\end{abstract}

Keywords: delay; energy efficient; MANETs networks; topology control algorithm; interference.

\section{INTRODUCTION}

An ad hoc wireless network is a special type of wireless networks that does not have a wired infrastructure to support communication among the wireless nodes. Each node acts as a router, as well as a communication end-point. There are many modern network applications that require QoS provisions in ad hoc networks, such as transmission of multimedia data, real-time collaborative work, and interactive distributed applications.

In order to provide the QoS requirement problem in terms of delay, some researchers found the delay incurred in a forwarding node or a routing path. [2] Delay depends on the speed of propagation and the number of hops a packet must travel to reach its destination that is one portion of the path between source and destination. Then, Xie et al. [3] said that in many cases the queuing delay takes a significant portion of the total delay over a hop. A path, which contains many packets in queue of the nodes and with short transmission delay on links, could have a larger delay than the one, which has less packets in the queue at nodes but longer transmission delay. And the larger the number of the intermediate nodes between the source and destination pair is, the larger the potential delay is. However, in wireless ad hoc networks, the impact of channel contention from neighbors must also be considered. Because of the limited channel source, access delay and collision are generated at nodes. If one of the nodes on a path can not acquire channel in a long period for contention, it may lead to massive packet drops and higher packet dropping rate. Processing delay and propagation delay which change in microseconds are much shorter than transmission delay, contention delay and queuing delay which change in millisecond. Therefore, the end-to-end (E2E) delay should consider: transmission delay over intermediate links, contention delay caused by nodes' contention for the shared channel and queuing delay induced at each intermediate node due to queuing policy or severe channel conditions.

Topology control is to dynamically change the nodes transmission range in order to maintain connectivity of the communication graph, while reducing energy consumption and/or interference that are strictly related to the nodes transmitting range. A good topology not only can provide a better service for routing layer, but also can save energy, increase network capacity and satisfy the QoS requirements. The previous topology control algorithms [4]-[7] mainly focused on the interference constraint. And how to employ topology control to reduce delay is not fully researched by those works. An alternative way to reduce the E2E delay is to increase the transmission power of a certain node in a path, so that the transmission range of the node is increased and thus the hops between the source and destination are reduced. Transmission delay may be decreased due to the reduction in hops; and the sum of the queuing delay along a path is also decreased because the number of the intermediate nodes is decreased. Thus, increasing the transmission power may reduce the E2E delay. However, it may cause more interference to other nearby active receiving nodes, excessive contention to nearby potential sending nodes, which may incur more retransmissions. And retransmission means the increase of E2E delay. Therefore, reducing delay and minimizing interference are two conflicting goals, and it is necessary to jointly consider a tradeoff between them. Thus, the problem of interference based topology control with delay-constraint is studied.

\section{RELATED WORK}

During developing real-time service like video, audio and voice over IP (VOIP), it is essential to provide efficient quality of service in MANET. In order to provide QoS to MANET, it should follow the standards of measure include bandwidth, hop count, delay, path reliability, energy consumption and service range. To achieve the above vision, it is significant to consider QoS routing in the QoS architecture. Delay requirement is one of the particularly useful QoS requirements for mobile ad hoc networks. Many QoS routing protocols which consider the end-to-end delay as a QoS measure have been proposed. Draves et al [2] defined the delay as the transmission delay of the packet. But it did not contain the queuing delay. If there are massive packets in the queue waiting for transmission since a node cannot transmit multiple packets simultaneously, the queuing delay 
may take a significant portion of the total delay. The delay in [7] contained the queuing delay and the transmission delay. But it did not explicitly consider the effects of channel contention. The contention of the channel can cause access delay and collision at Medium Access Control (MAC) layer.

\subsection{Topology Control}

\subsubsection{Taxonomy}

There are several different approaches to topology control and it is possible to organize them into a coherent taxonomy. The first distinction is between approaches that control transmitter power and those that impose a hierarchy in the network. Hierarchical approaches change the logical structure of the network in terms of node adjacencies and may be broken down into approaches that use clustering and those that use dominating sets.

The power control approaches act on the transmission power of nodes using several different techniques. The first distinction to make of power control approaches is between homogeneous and non-homogeneous approaches. Homogeneous topology control is the easier of the two in which nodes are assumed to use the same transmitting power and the problem of topology control becomes in essence one of finding the value of the transmitter range that satisfies a certain network wide property.

In non-homogenous topology control nodes are allowed to select different individual transmitting powers up to a certain maximum that they can support which means that they will have different transmitting ranges. This form of topology control can be split into three different categories according to the type of information that is used to generate the topology. These three categories are location based, direction based, and neighbor based.

In location-based approaches exact node locations are known and are either used by a centralized authority to calculate a set of transmitting range assignments which optimize a certain measure or are exchanged between nodes to create an approximately optimal topology in a distributed fashion. In direction-based approaches, nodes are assumed to not know their positions but can estimate the relative direction to each of their neighbors. Finally, in neighbor-based approaches the only knowledge nodes have of their neighbors is the neighbor IDs and the IDs are ordered according to some criterion when performing topology control.

\subsubsection{Quality Measures}

Different approaches to topology control will produce different results. For a collection of nodes, let denote the graph on for which there is an edge from node to node only if can directly reach. It is desirable to judge the usefulness of a topology returned by a topology control algorithm and compare it with results from other algorithms. In order to do this, some metrics and measures are required which include connectivity, energy efficiency, throughput, and robustness to mobility.

2.1.2.1 Connectivity: If there is a multihop path between and in, then there should also be a path in. This is a basic requirement for a topology control algorithm, that it should not disconnect a connected graph.

www.ijsea.com
2.1.2.2 Energy Efficiency: The energy consumed for a transmission between $\mathrm{u}$ and $\mathrm{v}$ is a polynomial function of the distance between $u$ and $v$. Two common notions of energy efficiency are the energy stretch factor and the hop stretch factor. The energy stretch factor is the worst increase in energy used to deliver a packet between any pair of nodes and along a minimum energy path between the original graph and the topology controlled graph G. The hop stretch is similar except that the focus is on path length as opposed to energy consumption. Formally

$$
\text { energy stretch factor }=\max _{u, v \in V} \frac{E_{T}(u, v)}{E_{G}(u, v)}
$$

(1) where is the energy consumed along the most energy efficient path in graph. Likewise

$$
\text { hop stretch factor }=\max _{u, v \in V} \frac{\left|(u, v)_{T}\right|}{\left|(u, v)_{G}\right|}
$$

(2) where is the shortest path in graph and is its length.

2.1.2.3 Node Degree: In order to better evaluate the performance of the topology control technique in terms of interference, a distinction is made between the physical and the logical node degree. The physical node degree refers to the number of neighbor nodes that are within the transmitter range of a given node. The logical node degree refers to the number of neighbor nodes that a given node is linked to.

2.1.2.4 Simplicity and Maintainability: It is desirable for a topology to be simple and easy to maintain and objective measures that can be used to evaluate these subjective goals are the number of edges in and the maximum node degree (number of neighbors) of any node in . It is desirable also for the algorithm used to have little overhead in terms of computation and communication requirements.

2.1.2.5 Throughput: It is desirable for the network topology to have a high throughput, where it is possible to sustain a comparable amount of traffic as the original network topology . Several throughput measures can be used [15] one of which is the bit-meter which is defined in terms of the bit-distance product. A network transports one bit-meter when it one bit is transported a distance of one meter. The throughput of the network is then the number of bit-meters transported per second.

2.1.2.6 Robustness to Mobility: The mobility of nodes causes neighborhood relationships to change in the original graph and some other nodes will have to change their topology information. A robust topology should only require a small number of these adaptations and avoid the effects of reorganization due to local node movement affecting the entire network. A measure of robustness is the adaptability which is the maximum.

\subsection{ITCD ALGORITHM}

ITCD Algorithm [1] describes a cross-layer distributed algorithm called interference-based topology control algorithm for delay-constrained (ITCD) MANETs with considering both the interference constraint and the delay constraint, which is different from the previous work. The transmission delay, contention delay and the queuing delay are taken into account in the proposed algorithm. Moreover, the impact of node mobility on the interference-based 
topology control algorithm is investigated and the unstable links are removed from the topology.

\subsubsection{Delay Model}

\subsubsection{Delay model of a path:}

The end-to-end delay contains transmission delay over intermediate links, contention delay caused by nodes' contention for the shared channel and queuing delay induced at each intermediate node due to queuing policy/or severe channel conditions. The transmission delay is the time for successful transmission, which is defined as the period from the instant that a packet is transmitted for the first time to the instant that it is either successfully transmitted or dropped after a predefined number of retransmissions. The contention delay is the access delay. And each retransmission will cause new access delay. The contention delay is determined by the contention window (CW) size of node, and the contention window size reflects the contention level. The queuing delay at an intermediate node can be interpreted as the interval between the time a packet reaches the node and the time this packet is to be transmitted.

For a path $\mathrm{P}: \mathrm{n} 1, \mathrm{n} 2, \ldots, \mathrm{ni}, \ldots, \mathrm{nN}(\mathrm{N} \geq 1)$, a packet is sent from node $\mathrm{n} 1$ to $\mathrm{nN}$ according to path $\mathrm{P} . \mathrm{L}(\mathrm{i})(\mathrm{i}+1)$ is the transmission delay of link between node $i$ and node $i+1$. Let $\mathrm{Ci}$ and Qi denote the contention delay and queuing delay at node $\mathrm{i}$, respectively. The total delay DP contains the contention delay and the queuing delay at each node and.The transmission delay of links on the path $\mathrm{P}$.

$$
D_{P}=\sum_{i=1}^{N-1}\left(L_{(i)(i+1)}+C_{i}+Q_{i}\right)
$$

\subsubsection{Delay model of an intermediate node:}

If the data is transmitted successfully in the first attempt, transmission delay of link between node $i$ and node $i+1$ is

$$
L_{(i)(i+1)}=\frac{L}{B}+D I F S+T_{A C K}+S I F S,
$$

where $\mathrm{L}$ denotes the packet length and $\mathrm{B}$ is transmission data rate, DIFS stands for the Distributed Inter-Frame Spacing, SIFS stands for the Short Inter-Frame Spacing and TACK represents the transmission delay of acknowledge frame.

\subsubsection{Delay constraint at an intermediate node:}

From the above equations, the delay constraint for a path is transformed into delay constraints at intermediate nodes. However, it is an extremely hard problem to partition the endto-end delay constraint into each node accurately. We define a max delay Dmax at intermediate nodes, which is similar to the max transmit power. Obviously, Dmax is determined by the information of the real-time requirement Treal and the number of hops $n$. Treal is given by the requirement of applications. As to $n$, we use a prediction method to estimate the number of hops.
Delay and topology control algorithm have been studied under the assumption that all nodes in the networks are stationary. Then the impact of mobility should be investigated. Firstly, our algorithm focuses on reducing a network interference to improve capacity while keeping connectivity and delay requirements. Most of the nodes have minimum connectivity. Frequent link breakages are prone to happen in the mobile environment. The poor links which are easily broken should be moved from the topology to reduce the effects of mobility. Secondly, if a receiving node moves around in a small area in the transmission range of the sender in a lower speed, delay at receiver is only determined by transmission delay, contention delay and queuing delay. If the node has a higher mobility, node may move out of the sender's transmission range quickly. The link between sender and receiver is unstable and prone to break. Once the link breaks, the transmission delay will become infinite. Thus, delay is also affected by mobility.

\subsection{SMART BOUNDARY GABRIEL GRAPH (SBYAOGG)}

YAO

The first objective was that it should be energy efficient and the second was that it should have low interference. To meet the set out design objectives, the routing sub graph $\mathrm{T}$ produced by the topology control technique from the original graph $\mathrm{G}$ had to meet certain requirements.

\subsubsection{Yao-Gabriel Graph With Smart Boundaries}

The algorithm is a mixture of the Gabriel graph algorithm and the Yao graph algorithm, with the use of smart region boundaries. The algorithm is referred to as the Smart Boundary Yao Gabriel Graph (SBYaoGG).

\section{ENERGY EFFICIENT, REDUCED DELAY AND MINIMUM DISTRIBUTED TOPOLOGY CONTROL ALGORITHM}

\subsection{Construction of EEDTCA}

A heuristic that was used whilst forming the reduced topology graph was to align the axis of the first cone used in the Yao graph computation to the region where nodes are most densely deployed. This can be accomplished by obtaining the unit direction vectors of all the neighboring nodes and then calculating the average direction vector. The average direction vector is then used as the axis of the cone for the first Yao graph region. The neighbor direction vectors and the average direction vector are illustrated in Fig. 1.

\subsubsection{Mobility}




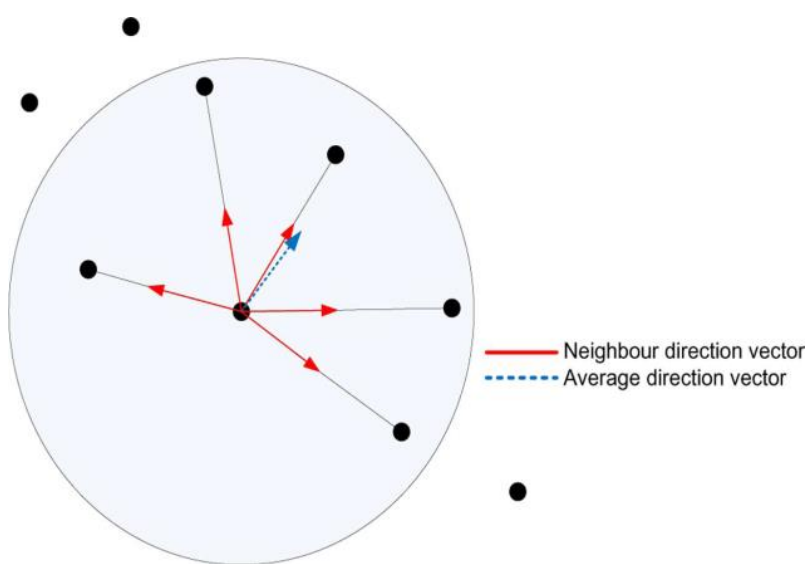

Figure 1 Neighbor direction vectors and the average direction vector.

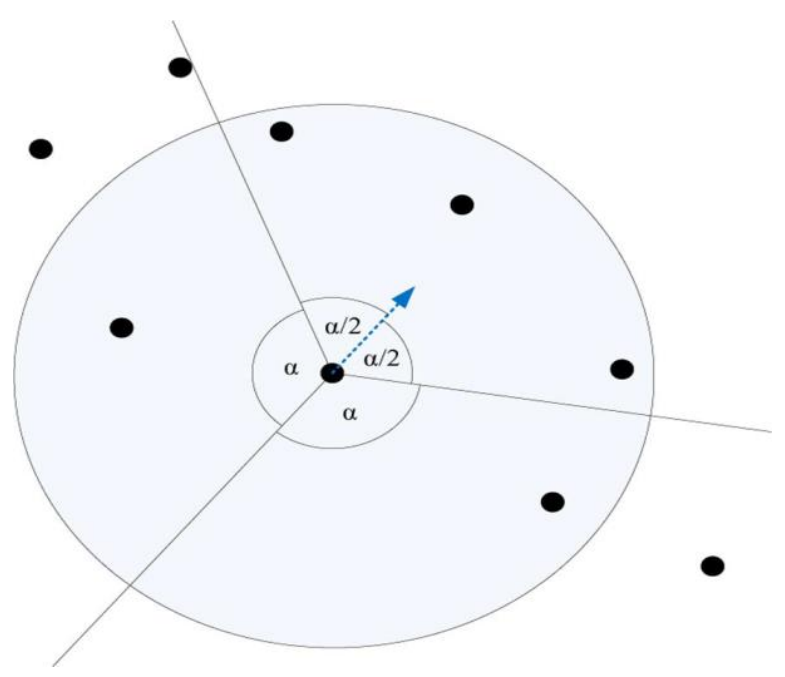

Figure 2 Graph boundaries using an average direction vector

\subsection{The Algorithm}

The following algorithm describes how to construct the EEDTCA, detailing the node in the network goes through.

Step1:The node discovers its neighbor nodes by broadcasting at maximum power.

Step2: The Gabriel graph is constructed locally.

Step3: The unit direction vectors of neighbor nodes in the Gabriel graph are computed.

Step4: The average direction vector is computed.

Step5: The axis of the cone of the first region to use in computing the Yao graph is set to correspond to the average direction vector.

Step6: The Yao graph is computed from the Gabriel graph, producing the reduced topology.

The final step in obtaining the EEDTCA is to optimize the reduced topology in order to ensure low interference and good power spanner properties. Two optimizations were made.

1) All edges are made symmetric by adding the reverse edge for any asymmetric link.

2) Transmitter power levels are set to the lowest level that will allow each node to reach all the nodes with which it has an edge.
After this the EEDTCA is fully formed and can be used as input to a routing algorithm.

\section{PERFORMANCE EVALUATION}

\subsection{Simulation Environment}

In order to evaluate the performance of the proposed EEDTCA protocol, we compare EEDTCA with the conventional ITCD [1] protocol and the energy-efficient and delay-constrained routing protocol which is to find an energyefficient path with explicit delay constraint. Adjusting the transmission power in data and set the pause-time to 0 . The simulation time for each simulation scenario is set to 50 seconds. In the results, each data point represents the average of 20 trials of experiments. The confidence level is $95 \%$, and the confidence interval is shown as a vertical bar in the figures. The detailed simulation parameters are shown in Table I.

The experiments are divided to three parts, and in each part we research the impact of one of the following parameters on the performance of routing protocols:

Table 1. Simulation parameters

\begin{tabular}{|c|c|}
\hline \multicolumn{2}{|c|}{ SIMULATION PARAMETERS } \\
\hline Simulation Parameter & NS-2(v2.34) \\
\hline Simulation Time & $50 \mathrm{~s}$ \\
\hline Topology Size & $1000 \mathrm{~m} * 1000 \mathrm{~m}$ \\
\hline Min speed & $0 \mathrm{~m} / \mathrm{s}$ \\
\hline Max speed & $2 \mathrm{~m} / \mathrm{s}$ \\
\hline Max nodes & 100 \\
\hline Pause time & $0 \mathrm{~s}$ \\
\hline Traffic Type & CBR \\
\hline Packet size & 512 bytes \\
\hline Mobility mode & Random way point \\
\hline
\end{tabular}

Number of nodes: We vary the number of nodes from 50 to 100 in a fixed field to research the impact of different network density. In this part, we set the number of CBR connections to 10.

Number of CBR connections: We vary the number of randomly chosen CBR connections from 2 to 10 with a fixed packet rate to research the impact of different traffic load. In this part, we set the number of nodes to 100 .

Delay constraint: We vary the delay constraint from $40 \mathrm{~ms}$ to $140 \mathrm{~ms}$ in a fixed field to research the impact of delay constraint. In this part, we set the number of nodes to 100 , the number of CBR connections to 15 .

\subsection{Performance with Varied Number of Nodes}




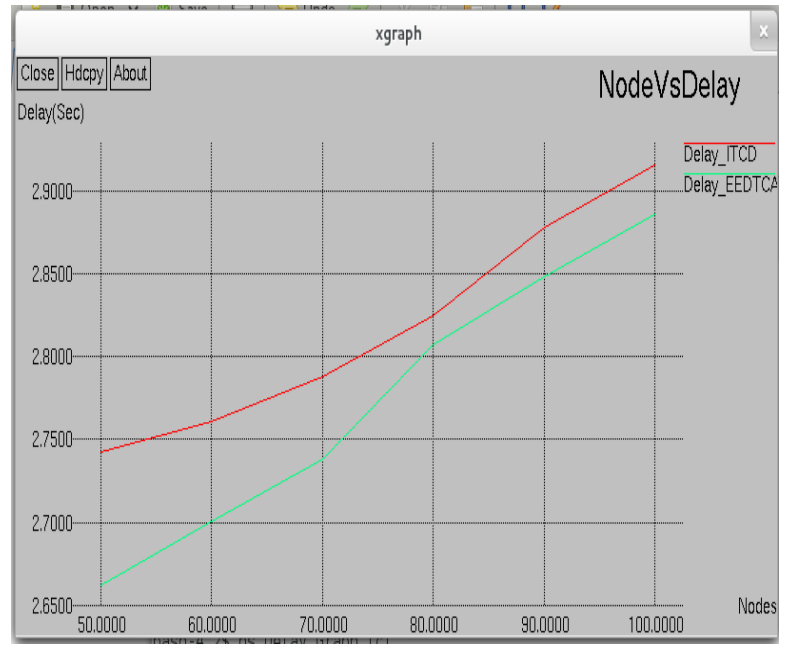

Figure3. Performance of delay with varied Number of Nodes.

Figure. 3 measures the average end-to-end delay of CBR packets received at the destinations with increasing network density. Average delay is defined as the average delay of a successfully delivered CBR packet from the source node to the destination node. In MANETs, inappropriate transmission power will increase the delay. If transmission power is too large, it will incur too many channel contentions, which increases the backoff timer in MAC layer, so as to aincrease the delay. EEDTCA protocol shows minimum delay while compairing with ITCD.

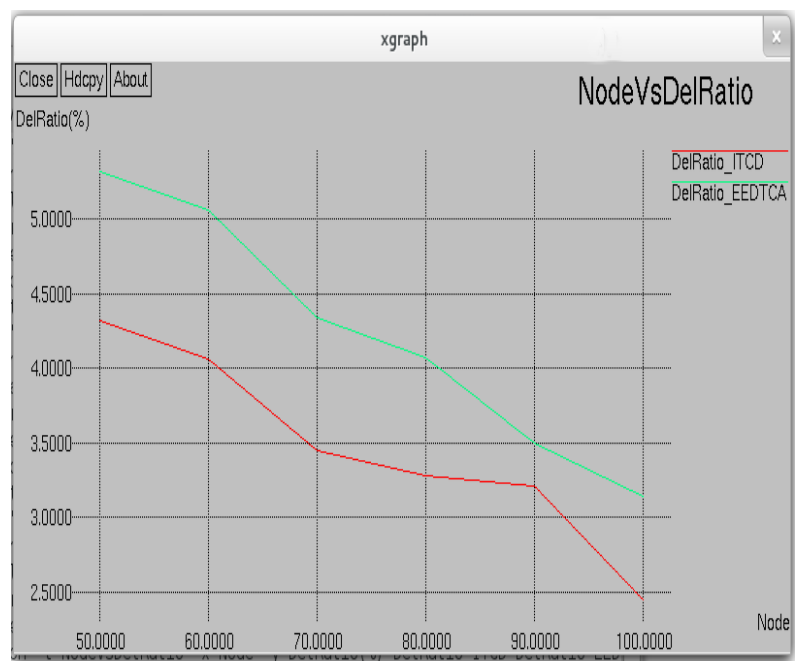

Figure4. Packet delivery ratio with varied number of Nodes.

Figure. 4 shows the packet delivery ratio with increasing network density. Hence, the EEDTCA protocol can increase the packet delivery ratio. On average, the packet delivery ratio is improved in the EEDTCA protocol when compared with the conventional ITCD protocol.

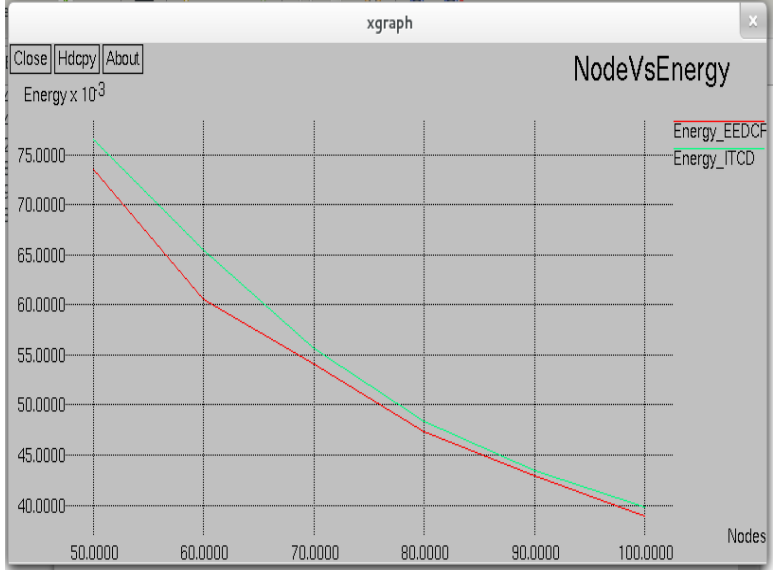

Figure5. Performance with Varied Energy with Node

Figure. 5 describes the energy consumption in the network with increasing the node density. The energy cosumption of EEDTCA protocol is lower while comparing with the existing ITCD routing protocol.

\subsection{Performance with Varied Number of CBR Connections}

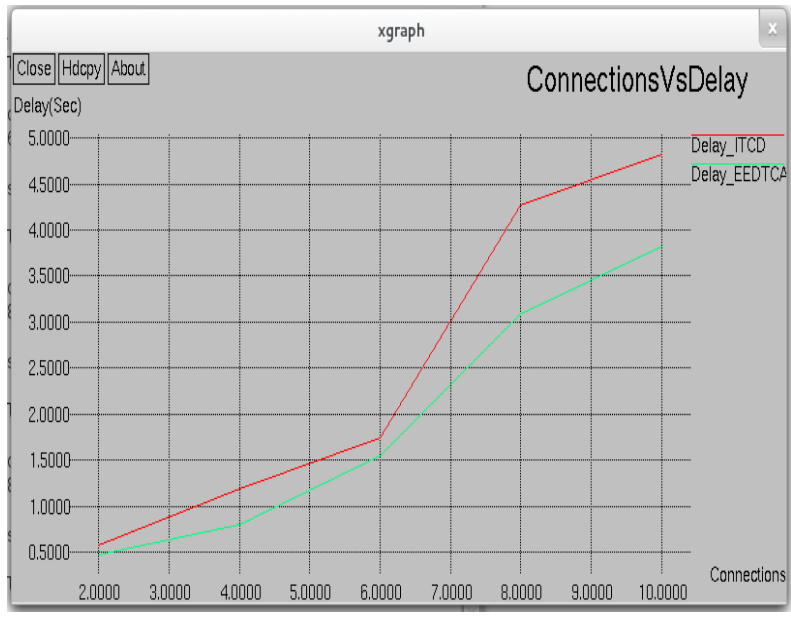

Figure 6. Performance of Delay with varried network traffic (ms)

Fig. 6 measures the average end-to-end delay of CBR packets received at the destinations with increasing traffic load. On average, the end to-end delay is reduced in the EEDTCA protocol when compared with the conventional ITCD protocol while increasing the traffic load. 


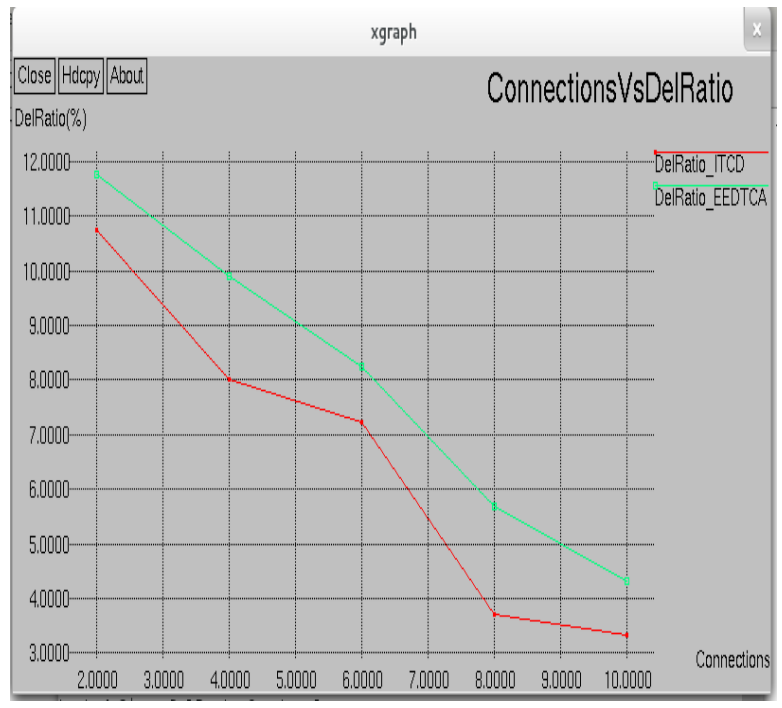

Figure 7. Performance of Packet Delivery Ratio with varried network traffic (ms)

Fig. 7 shows the packet delivery ratio with increasing network traffic. The EEDTCA protocol can increase the packet delivery ratio. The packet delivery ratio is improved in the EEDTCA protocol when compared with the conventional ITCD protocol.

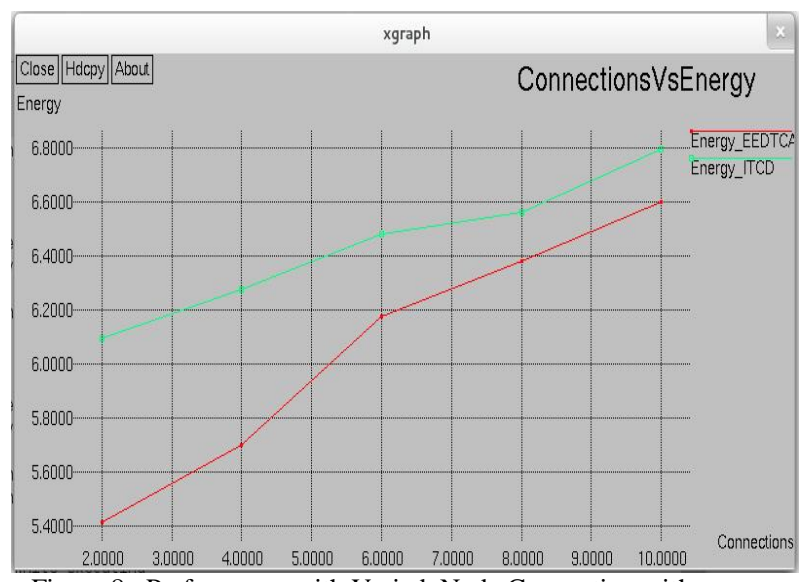

Figure 8. Performance with Varied Node Connection with energy

Fig. 8 describes the energy consumption of EEDTCA protocol. The energy cosumption of EEDTCA protocol is low while comparing with the existing ITCD routing protocol.

\section{CONCLUSION}

In this paper, we propose energy efficient, reduced delay and minimum distributed topology control algorithm for mobile ad hoc networks. The simulation results show that EEDTCA can reduce the delay and improve the packet delivery performance effectively with efficient energy in mobile ad hoc networks. In future we can study behavior of this algorithm for senor networking environment

\section{REFERENCES}

[1] Xin Ming Zhang, Member, IEEE, Yue Zhang, Fan Yan, Athanasios V.Vasilakos, Senior Member, IEEE, April 2015, Interference-Based Topology Control Algorithm for Delay-Constrained Mobile Ad Hoc Networks.
[2] Steve A. Borbasb and Esther H. Jennings, Neural Networks, 2002. IJCNN '02. Proceedings of the 2002 International Joint Conference on 2002, Distributed Topology Control Algorithm for Multihop Wireless Networks.

[3] F. Xie, X.M. Zhang, J.F. Guo, and G.L. Chen, "A delay oriented adaptive routing protocol for mobile ad hoc networks,(in Chinese with English abstract)," Journal of Software, vol. 16, no. 9, pp. 1661-1667, 2005.

[4] N. Burri, P.V. Rickenbach, R.Wattenhofer and Y. Weber, " Topology control made practical: increasing the performance of source routing," in Proc. 2nd International Conference on Mobile Ad-hoc and Sensor Networks, pp. 1-12, 2006

[5] Forman, G. 2003. An extensive empirical study of feature selection metrics for text classification. J. Mach. Learn. Res. 3 (Mar. 2003), 1289-1305.

[6] Brown, L. D., Hua, H., and Gao, C. 2003. A widget framework for augmented interaction in SCAPE.

[7] M. Kadivar, M.E. Shiri, and M. Dahghan, "Distributed topology control algorithm based on one- and two-hop neighbors' information for ad hoc networks," Computer Communications, vol. 32, no.2, pp. 368-375, 2009.

[8] Spector, A. Z. 1989. Achieving application requirements. In Distributed Systems, S. Mullender 